\title{
Technical Note: A data-driven method for estimating the composition of end-members from streamwater chemistry observations
}

\author{
Esther Xu Fei ${ }^{1}$ and Ciaran Joseph Harman ${ }^{1,2}$ \\ ${ }^{1}$ Department of Environmental Health and Engineering, Johns Hopkins University \\ ${ }^{2}$ Department of Earth and Planetary Science, Johns Hopkins University \\ Correspondence: Ciaran Joseph Harman (charman1@jhu.edu)
}

\begin{abstract}
End-Member Mixing Analysis (EMMA) is a method of interpreting streamwater chemistry variations and is widely used for chemical hydrograph separation. It is based on the assumption that the streamwater is a mixture of varying contributions from relatively time-invariant source solutions (end-members). These end-members are typically identified by collecting additional measurements of candidate end-members from within the watershed. This technical note introduces a complemen-

5 tary, data-driven method: Convex-Hull End-Member Mixing Analysis (CHEMMA), to infer the end-member compositions and their associated uncertainties from the streamwater observations alone. The method involves two steps. The first step uses Convex-Hull Non-negative Matrix Factorization (CH-NMF) to infer possible end-member compositions by searching for a simplex that optimally encloses the streamwater observations. The second step uses Constrained K-means Clustering (COP-KMEANS) to classify the results from repeated applications of CH-NMF to analyze the uncertainty associated with the algorithm. In an example application using the 1986 to 1988 Panola Mountain Research Watershed dataset, CHEMMA is able to robustly reproduce the three field-measured end-members found in previous research using only the streawater chemical observations. It also suggests the existence of a fourth end-member. Further work is needed to explore the constraints and capabilities of this approach.
\end{abstract}

\section{Introduction}

End-Member Mixing Analysis (EMMA) has been used to interpret observed streamwater chemical concentration profile variability in terms of time-varying contributions from end-member "sources", each supplying water with a constant concentration profile. This method has been applied in many different hydro-climatic and geology settings (Bernal et al., 2006; Hooper et al., 1990; Li et al., 2019; Lv et al., 2018; Jung et al., 2009; Neill et al., 2011). EMMA has also been used to distinguishing sources of dissolved organic matter in natural streams (Hur et al., 2006; Yang and Hur, 2014), specific conductance (Kronholm and Capel, 2015), and other combinations of streamwater attributes that can be assumed to have conservative mixing (Barthold 
et al., 2011).

EMMA assumes that the chemical composition of streamwater should be explained by the conservative mixing of a finite set of temporally invariant end-members (Hooper et al., 1990). These end-members, therefore, are the most extreme points that define a range within which all streamwater observations are included. End-members are identified by collecting samples of candidate source-water within the watershed. Inasmuch as the end-members are identified by candidate sampling, they depend upon hypotheses that 1) identified end-members supply streamwater; and 2) identified end-member set may be incomplete.

Streamwater concentration are naturally correlated. EMMA utilizes the Principal Component Analysis (PCA) method to convert the naturally correlated streamwater concentrations into a set of linearly uncorrelated variables. Each new variable, which is called Principal Component (PC), is a linear combination of the observed streamwater attributes. For a set of $n$ variables, PCA first requires standardized observations $\left(\mathbf{X}_{o b s}\right)$ by subtracting the mean and dividing the standard deviation. Then it calculates a projection matrix $\mathbf{P}_{o b s}$, which transforms the from the observation space to the PC space, by decomposing the covariance matrix of $\mathbf{X}_{o b s}$. The transformed columns of $\mathbf{Y}_{o b s}$ (representing the $n$ observations in the PC space) are uncorrelated, and each of which accounts for a portion of total variance:

$\mathbf{Y}_{o b s}=\mathbf{X}_{o b s} \mathbf{P}_{o b s}^{T}$.

Standardized end-member candidates $\mathbf{X}_{e m}$ can be projected into the PC space by the same projection matrix $\mathbf{P}_{o b s}$, and be converted in the transformed space as $\mathbf{Y}_{e m}$ :

$40 \quad \mathbf{Y}_{e m}=\mathbf{X}_{e m} \mathbf{P}_{o b s}^{T}$.

To find the parsimonious subset of appropriate end-members, EMMA then takes the information provided by PCA to determine the approximate dimensionality of the streamwater mixture and to screen end-members. In the PC space, appropriate end-member candidates $\left(\mathbf{Y}_{\text {em }}\right)$ are selected by choosing ones that tightly bound the transformed observations $\left(\mathbf{Y}_{\text {obs }}\right)$ (Christophersen and Hooper, 1992; Hooper et al., 1990; Hooper, 2003). However, the number of retained PCs is usually determined using a heuristic, such as using the number of PCs that explain at least $\frac{1}{n}$ proportion of the total variance, because of the need to capture the variance. After subjectively determined the number of PCs, Christophersen and Hooper (1992) mathematically proved that one end-member more than the number of PCs is required to describe the rank of the streamwater observation.

There are limitations to this approach, that can result in spurious or incomplete source identification (Delsman et al., 2013; 50 Hooper, 2003; Valder et al., 2012; Yang and Hur, 2014). Specifically, 1) the composition of a source cannot be determined unless candidate end-member measurements are obtained that are representative of it; 2) determining the number of significant PC is subjective; 3) EMMA is not able to deal with non-conservative mixing if non-linear structure are not provided to replace the current simplex structure (Christophersen and Hooper, 1992). 

et al., 2001; Delsman et al., 2013; Hooper and Christophersen, 1992; James and Roulet, 2006; Jung et al., 2009; Li et al., 2019; Lv et al., 2018; Neal et al., 1992; Neill et al., 2011; Valder et al., 2012), there is not a method to characterize missing or unmeasured end-members. In this technical note, we focus on improving the identification of end-member compositions and associated uncertainties. The method depends on the idea inherited from EMMA that the end-members are located near the most extreme points that bound the observations in "mixing" space. This suggests that we might be able to interrogate the observational data projected in the end-member space to find such extremal points as end-members (even if no individual samples fully represent that end-member). We propose a new EMMA approach, Convex-Hull End-Member Mixing Analysis (CHEMMA), which is a data-driven method that characterizes the end-member chemical compositions, as well as an intrinsic uncertainty component associated with the end-member. The ability of the method to infer end-members is demonstrated by an application to the 1986 to 1988 Panola Mountain Research Watershed dataset published in Hooper and Christophersen (1992).

\section{Methodology}

Convex-Hull End-Member Mixing Analysis (CHEMMA) applies a matrix factorization method, Convex-Hull Non-negative Matrix Factorization (CH-NMF), along with a classification method, Constrained K-means Clustering (COP-KMEANS), to find end-member compositions under EMMA assumptions. The CH-NMF method provides a numerical iterative algorithm to search for end-member compositions that optimally enclose the streamwater observations in the PC space. The $\mathrm{CH}-\mathrm{NMF}$ algorithm is run many times because each iteration of the search can result in highly non-unique optima. We apply the COPKMEANS method to classify the CH-NMF numerical outputs into clusters. The centroid of each cluster is assumed to represent our best estimate of an end-member.

\subsection{Adaption of CH-NMF to the EMMA problem}

The concepts of "convex combination" and "convex hull" connect CH-NMF with the idea of end-member mixing. A convex combination is equivalent to a weighted sum. It is a linear combination of vectors where the weight associated with each vector varies between zero to one, and the weights sum to one. If we construct a simplex, which means a highly dimensional polytope, with some distinct vectors at its vertices, this simplex is a convex hull that encloses points within the hull to be a convex combination of the vertices. Similarly, if we conservatively mixed distinct end-members, the streamwater chemical concentration observations can be a weighted sum of end-members with their contributions. The ideas of "convex combination" and "convex hull" are mathematically identical to the end-member mixing.

The CH-NMF method describes a general methodology of finding the most extreme points (end-members) that form a simplex with $\mathrm{k}$ vertices around the $n$-dimensional observation data cloud by searching for convex hull that enclose the data when projected into a linear lower dimensional projection subspaces (Thurau et al., 2011). First, the observations are standardized (zero mean and unit variance), the PC vectors are calculated, and the top $k$ PCs are retained as with EMMA. Next, the stan- 
dardized data are projected into the 2D subspace spanned by two of the PCs. Qualified points forming a convex hull around the projected data are marked. This is repeated for every pair of PCs. Finally, we interpolate between convex-hull vertices in each subspace to find the vertices of a simplex in a $k$-dimensional subspace. This simplex forms a "convex-hull" such that all the data points can be optimally approximated as convex linear combinations of them. The algorithm is summarized as follows:

Algorithm 1: CH-NMF algorithm (Thurau et al., 2011) adapted to the end-member identification problem given $m$ streamwater observations of $n$ solutes

Result: $i^{t h}$ end-member composition $\boldsymbol{x}_{e m i}^{n \times 1}$, and its contribution $\boldsymbol{h}_{i}^{m \times 1}, i=1,2, \ldots, k$

1. Subtract the mean $\left(\mu_{1: n}\right)$ and dividing by standard deviation $\left(\sigma_{1: n}\right)$ for each solute to obtain standardized observation matrix $\mathbf{X}_{o b s}^{m \times n}$

2. Compute $d$ eigenvectors (PCs) $\boldsymbol{e}_{1}, \ldots, \boldsymbol{e}_{d}, d=\operatorname{rank}\left(\mathbf{X}_{o b s} \mathbf{X}_{o b s}^{T}\right) \leq n$

3. Project $\mathbf{X}_{\text {obs }}$ onto each of the $\left(\begin{array}{l}d \\ 2\end{array}\right)$ 2D-subspaces spanned by pairs of PCs

4. Mark the $k$ convex hull vertices for each projection plane and stored in matrix $\mathbf{S}^{n \times p}, p$ is the maximum number of points needed to make a convex hull in one projection plane.

5. Define end-member matrix $\mathbf{X}_{e m}^{n \times k}=\left[\boldsymbol{x}_{e m 1}, \boldsymbol{x}_{e m 2}, \ldots, \boldsymbol{x}_{e m k}\right]$ and let $\mathbf{X}_{e m}=\mathbf{S I}$, $\operatorname{minimize}\left\|\mathbf{S}-\mathbf{S I}^{p \times k} \mathbf{J}^{k \times p}\right\|_{F}^{2}$, s.t. $\sum_{i} \boldsymbol{i}_{j}=1, i_{i j} \in[0,1]$, and $\sum_{i} \boldsymbol{j}_{j}=1, j_{i j} \in[0,1]$

6. Minimize $\left\|\mathbf{X}_{o b s}-\mathbf{H}^{m \times k} \mathbf{X}_{e m}^{T}\right\|_{F}^{2}$, s.t. $\sum_{j} \boldsymbol{h}_{\boldsymbol{i}}=1, h_{i j} \in[0,1]$

With given standardized $m$ streamwater samples with $n$ measured attributes $\mathbf{X}_{o b s}^{m \times n}$ and desired $k$ end-members (Step 1, Figure $1 \mathrm{a}$ ), CH-NMF decomposes the covariance matrix of the observations to obtain $d$ PCs, which is the same linear orthogonal projection as PCA method (Step 2). Instead of immediate dimension reduction as EMMA, CH-NMF examines the distribution of $\mathbf{X}_{o b s}$ in all of the subspaces spanned by PC pairs (Step 3, Figure $1 \mathrm{~b}$, light blue points) and marks the most extreme points (Figure $1 \mathrm{~b}$, red crosses) that construct the convex hull (Figure $1 \mathrm{~b}$, red lines) to store in $\mathbf{S}$ (Step 4). Then, a subset of $\mathbf{S}, \mathbf{S I}=\mathbf{X}_{e m}$, is found as a convex combination of $\mathbf{S}$ (Step 5, Figure $1 \mathrm{c}$, square vertices of the simplex) that minimizes the Frobenius norm $\|\cdot\|_{F}^{2}$ (the entry-wise Euclidean norm of the matrix). Finally, the contribution $\mathbf{H}$ is found by finding the convex combination of end-members that reproduces the data with minimal error (again using the Frobenius norm) (Step 6).

Step 5 is the essential step of the CH-NMF theory, and it is a modification of Convex Nonnegative Matrix Factorization (C-NMF) by adding a convexity constraint on $\mathbf{J}$ (Ding et al., 2008; Thurau et al., 2011). In the original setting of C-NMF, the $\mathbf{I}$ and $\mathbf{J}$ are naturally sparse if the vertex search is in PC subspaces (Ding et al., 2008). Adding the convexity constraint on $\mathbf{J}$ makes $\mathbf{J}$ an interpolation between each columns of $\mathbf{S I}$ (i.e. each end-member composition $\boldsymbol{x}_{e m}$ ), however, the sparse nature of I remains (Thurau et al., 2011). 
We could interpret the objective function of Step 5 (minimize $\left\|\mathbf{S}-\mathbf{S I}^{p \times k} \mathbf{J}^{k \times p}\right\|_{F}^{2}$ ) in three steps. First, the sparsity of $\mathbf{I}$ results in the end-member composition $\mathbf{X}_{e m}$ close to a subset of the extreme observations (S) projected in the PC subspace. Second, $\mathbf{J}$ makes other extreme observations in $\mathbf{S}$ to be expressed as a convex combination (interpolation) of $\mathbf{X}_{e m}$. Third, minimizing the Frobenius distance between $\mathbf{S}$ and $\mathbf{X}_{e m} \mathbf{J}$ guarantees end-member compositions $\mathbf{X}_{e m}$ will be convex hull vertices because all other extreme points can be written as convex combinations of vertices, but not vice versa. As a consequence, a well-supported set of convex hull vertices tightly bound the observations and are as unique as possible, which satisfies the original EMMA assumption of finite set of distinct end-members. The sparse nature of $\mathbf{I}$ helps prevent overfitting because noise will tend to be concentrated on superfluous vertices without degrading identification of the others. The noisy end-members can be identified in the classification step given in the next section.

The constraint requiring that the end-members be a convex combination of the extreme observations implies that CH-NMF may not accurately identify end-members that are not a large fraction of any observation in the dataset. As the synthetic example shown in Figure 1 illustrates, the simplex formed by joining the CH-NMF end-members lies inside the shell formed by connecting the extreme points (red crosses in Figure 1c). If no samples are anywhere close to being 'pure' representatives of an end-member, the apparent end-member identified by CH-NMF may lie closer to the data centroid than the true endmember. Methods to relax the constraint on Step 5 and better identify end-members distant from the data in mixing space will be investigated in future work.

\subsection{Quantify the intrinsic uncertainty using COP-KMEANS}

Each run of CH-NMF may yield different end-member estimates. This is because the complex structure of the high-dimensional streamwater data result in a rough objective function surface (Step 5). CH-NMF runs with different initial search locations may fall into different local minima.

Depending on the structure of the data cloud, each run's end-members may be nearly identical (if the end-member is wellcharacterized) or one or more may vary widely. Poor identification of extreme points may result from a lack of sufficient well-defined "vertices" in the data cloud. This may occur if more end-members are sought than the data can support. It may also occur if an end-member is variable in time. Instead of a vertex, the time-varying end-member forms an edge in the data space. Alternatively, the observations may not sample the true mixing space sufficiently to identify an end-member in the space as a convex-hull vertex, perhaps because it never represents more than a small fraction of variance.

Even in the absence of these issues, the variability and uncertainty of the stream concentration observations will contribute to uncertainty in end-member identification. The variation in the CH-NMF-identified end-members can be assessed by running the $\mathrm{CH}-\mathrm{NMF}$ analysis a large number of times, and then using a clustering algorithm to extract the centroid and spread of areas consistently identified as an end-member. We use the COP-KMEANS variant of the K-means clustering algorithm, which allows us to require that end-members predicted from the same $\mathrm{CH}-\mathrm{NMF}$ run must not be placed in the same cluster 
(Wagstaff et al., 2001). This is achieved by assigning a "cannot-link" constraint between every pair of candidate end-members generated by the same CH-NMF run. Apart from the "cannot-link" constraints, COP-KMEANS works identically to normal k-means clustering (Wagstaff et al., 2001). For each cluster identified by COP-KMEANS, we can qualitatively examine the spatial distribution of the associated end-members, and quantitatively calculate the centroid and variance of the cluster.

As the number of end-members increases, the centroid and variance within the cluster may increase or decrease, which provides another way to decide the number of needed end-members for a given observation set. In this technical note, we consider a new cluster to be well-identified as a proper end-member if two conditions are both satisfied: 1) the spread of previously identified clusters remains similar or decreases, and 2) the cluster itself has a reasonable variance.

\subsection{Example Python implementation}

An example Python implementation of CHEMMA including the application to Panola Mountain data presented in the next section are available in a Jupyter Notebook on GitHub (https://github.com/Estherrrrrxu/CHEMMA). The CH-NMF section uses a Python package, pymf.chnmf, detailed in Thurau et al. (2011). The COP-KMEANS section uses a Python package, COP-Kmeans presented in Babaki (2017).

\section{An application of CHEMMA}

We applied CHEMMA to a test dataset of 905 samples of six solutes (alkalinity, sulfate, sodium, magnesium, calcium, and dissolved silica) collected from the stream in the Panola Mountain research catchment, Georgia, U.S. and described in Hooper et al. (1990). The six solutes were specifically selected to meet EMMA's assumption that their concentrations vary significantly across the watershed (Hooper et al., 1990). Hooper et al. (1990) found that the stream chemistry could be interpreted as a mixture of hillslope, groundwater, and organic soil end-members, which are identified by sampling within the watershed. Here we ask 1) does CHEMMA recover the same three end-members as Hooper et al. (1990) identified in field-sampling? and 2) does the data support the existence of additional end-members?

We ran CHEMMA for three, four, and five end-member cases $(k=3,4,5)$ because two and three PCs account for $94 \%$ and $97 \%$ of the total variance, respectively. In order to capture the intrinsic uncertainty associated with the identified clusters, we calculated the mean and standard deviation (st.dev) for each case based on $100 \mathrm{CH}-\mathrm{NMF}$ runs (Table 1). CHEMMA was able to recover the three field-measured end-members reported by Hooper et al. (1990) (Figure 2, three blue stars). The mean of the three CHEMMA identified clusters (Figure 3 and Table 1) are very similar to the median concentration of the field-measured end-members (Table 2). The median concentration of the hillslope field sample (Table 2) has much lower alkalinity concentration compared with the mean concentration of the CHEMMA identified Green cluster (Figure 3 and Table 1), however, it is still within the cluster spread given in Table 1. 
A fourth end-member could be robustly identified (Figure 2, four red stars) that explained more of the data variability. This end-member apperared to be a mixture of hillslope and groundwater in some ways but had relatively high alkalinity and silica concentration compared to those end-members (Figure 2 brown and navy axes). The fourth end-member captures variations along the third PC axis (Figure $3 \mathrm{~d}$ ), which are not apparent in the $2 \mathrm{D}$ view (Figure $3 \mathrm{~b}$ ).

The spread of all end-member clusters (generated by 100 runs of CH-NMF) was small when four were sought, but a fifth could not be clearly identified. As the number of end-members was increased from three (Figure 3 a) to four (Figure 3 b), the new cluster (cyan Cluster 4) was dense, while the other three clusters (green, blue, and red) remained at similar locations to those clusters identified in the three end-member case. Adding the fourth end-member reduced the spread of the previously identified three clusters in the PC subspace (Figure 3 a and b and Table 1) suggesting they could now be identified with less uncertainty. However, the inclusion of the fifth end-members (Figure $3 \mathrm{c}$ ) not only did not further tighten the previously identified clusters, but the fifth cluster was poorly defined (black Cluster 5). Except the cyan cluster has generally decreased within cluster variation, the standard deviations of other clusters increase for both three and four end-member cases (Table 1).

\section{Discussion and conclusions}

As the application results show, CHEMMA is able to reproduce the three end-members that were verified in the previous study as well as a fourth end-member that explains more variation in the data (Hooper et al., 1990). The dispersed cluster distributions in Figure $3 \mathrm{c}$ suggests that the fifth end-member may be finding the noisy edges of the sample space, and so cannot be supported by the data. However, we have not here identified an objective criteria for determining whether an end-member is supported.

Because CHEMMA extracts end-members from the observations, the accuracy of the end-member's composition is influenced by the noise from sample chemical analysis error, how well the collected samples represent the full range of sources in the catchment, and how valid the assumption of conservatively-mixing time-invariant end-members is. The captured variations in PC 3 shown in Figure $3 \mathrm{~d}$ may result from temporal variations of the end-member composition. The less concentrated Cluster 3 in Figure 3 b may result from relatively rare contributions from that end-member. Fortunately, CHEMMA itself provides a tool for exploring some of these sources of uncertainty. By partitioning the dataset into time periods (or hydrologic state, etc), the temporal variability of end-members could be explored.

Future work refining and applying this method may focus on 1) applying quantitative methods to optimize the model complexity, such as the Akaike Information Criterion (AIC), or Bayesian Information Criterion (BIC, or Schwarz criterion); 2) relaxing the constraints on the $\mathrm{CH}-\mathrm{NMF}$ algorithm (Algorithm 1, Step 5) so that extreme points in $\mathbf{S}$ also lie inside the simplex, allowing the method to better characterize end-members that are never a large fraction of any observations; and 3) exploring 
https://doi.org/10.5194/hess-2020-250

Preprint. Discussion started: 15 June 2020

(c) Author(s) 2020. CC BY 4.0 License.

205 the data requirements and uncertainty of the method, including better understanding the relationship between the stability of COP-KMEANS clusters, the temporal variability of end-members, and the number of samples.

Code and data availability. Both the example code and data are available in a Jupyter Notebook on GitHub https://github.com/Estherrrrxu/ CHEMMA (Xu Fei, 2020).

Author contributions. Xu Fei and Harman were responsible for conceptualization, methodology, and visualization. Xu Fei was responsible

210 for investigation, formal analysis, and writing (original draft). Harman was responsible for funding acquisition, supervision, and writing (review \& editing).

Competing interests. The authors have no competing interests to declare.

Acknowledgements. Panola stream solute chemistry data from Hooper and Christophersen (1992) was collected with the support of the USGS and is available at http://hiscentral.cuahsi.org/pub_network.aspx?n=385. Thanks to Rick Hooper for providing thoughtful feedback on the draft manuscript. This work was supported by NSF grant EAR-1654194. 


\section{References}

Ali, G. A., Roy, A. G., Turmel, M. C., and Courchesne, F.: Source-to-stream connectivity assessment through end-member mixing analysis, Journal of Hydrology, 392, 119-135, https://doi.org/10.1016/j.jhydrol.2010.07.049, 2010.

Babaki, B.: COP-Kmeans version 1.5, https://github.com/Behrouz-Babaki/COP-Kmeans, https://doi.org/10.5281/zenodo.831850, 2017.

Barthold, F. K., Tyralla, C., Schneider, K., Vaché, K. B., Frede, H.-G., and Breuer, L.: How many tracers do we need for end member mixing analysis (EMMA)? A sensitivity analysis, Water Resources Research, 47, 1-14, https://doi.org/10.1029/2011WR010604, 2011.

Bernal, S., Butturini, A., and Sabater, F.: Inferring nitrate sources through end member mixing analysis in an intermittent Mediterranean stream, Biogeochemistry, 81, 269-289, https://doi.org/10.1007/s10533-006-9041-7, 2006.

Burns, D. A., Mcdonnell, J. J., Hooper, R. P., Peters, N. E., Freer, J. E., Kendall, C., and Beven, K.: Quantifying contributions to storm runoff through end-member mixing analysis and hydrologic measurements at the Panola Mountain Research Watershed ( Georgia, USA), Hydrological Processes, 15, 1903-1924, https://doi.org/10.1002/hyp.246, 2001.

Christophersen, N. and Hooper, R. P.: Multivariate analysis of stream water chemical data: the use of Principal Components Analysis for the end-member mixing problem, Water Resources Research, 28, 99-107, 1992.

Delsman, J. R., Oude Essink, G. H., Beven, K. J., and Stuyfzand, P. J.: Uncertainty estimation of end-member mixing using generalized likelihood uncertainty estimation (GLUE), applied in a lowland catchment, Water Resources Research, 49, 4792-4806, https://doi.org/10.1002/wrcr.20341, 2013.

Ding, C. H., Li, T., and Jordan, M. I.: Convex and semi-nonnegative matrix factorizations, IEEE transactions on pattern analysis and machine intelligence, 32, 45-55, 2008.

Hooper, R. P.: Diagnostic tools for mixing models of stream water chemistry, Water Resources Research, 39, 1055, https://doi.org/10.1029/2002WR001528, 2003.

Hooper, R. P. and Christophersen, N.: Predicting episodic stream acidification in the southeastern United States: combining a long-term acidification model and the end-member mixing concept, Water Resources Research, 28, 1983-1990, https://doi.org/10.1029/92WR00706, 1992.

Hooper, R. P., Christophersen, N., and Peters, N. E.: Modelling streamwater chemistry as a mixture of soilwater end-members - an application to the Panola Mountain Catchment, Georgia, U.S.A., Journal of Hydrology, 116, 321-343, 1990.

Hur, J., Williams, M. A., and Schlautman, M. A.: Evaluating spectroscopic and chromatographic techniques to resolve dissolved organic matter via end member mixing analysis, Chemosphere, 63, 387-402, https://doi.org/10.1016/j.chemosphere.2005.08.069, 2006.

James, A. L. and Roulet, N. T.: Investigating the applicability of end-member mixing analysis (EMMA) across scale: A study of eight small, nested catchments in a temperate forested watershed, Water Resources Research, 42, 1-17, https://doi.org/10.1029/2005WR004419, 2006.

Jung, H. Y., Hogue, T. S., Rademacher, L. K., and Meixner, T.: Impact of wildfire on source water contributions in Devil Creek, CA: evidence from end-member mixing analysis, Hydrological Processes, 23, 183-200, https://doi.org/10.1002/hyp, 2009.

Kronholm, S. C. and Capel, P. D.: A comparison of high-resolution specific conductance-based end-member mixing analysis and a graphical method for baseflow separation of four streams in hydrologically challenging agricultural watersheds, Hydrological Processes, 29, 25212533, https://doi.org/10.1002/hyp.10378, 2015.

Li, X., Ding, Y., Han, T., Kang, S., Yu, Z., and Jing, Z.: Seasonal controls of meltwater runoff chemistry and chemical weathering at Urumqi Glacier No.1 in central Asia, Hydrological Processes, 33, 3258-3281, https://doi.org/10.1002/hyp.13555, 2019. 
https://doi.org/10.5194/hess-2020-250

Preprint. Discussion started: 15 June 2020

(c) Author(s) 2020. CC BY 4.0 License.

Lv, Y., Gao, L., Geris, J., Verrot, L., and Peng, X.: Assessment of water sources and their contributions to streamflow by end-member mixing analysis in a subtropical mixed agricultural catchment, Agricultural Water Management, 203, 411-422, https://doi.org/10.1016/j.agwat.2018.03.013, 2018.

Neal, C., Robson, A., Reynolds, B., and Jenkins, A.: Prediction of future short-term stream chemistry - a modelling approach, Journal of Hydrology, 130, 87-103, https://doi.org/10.1016/0022-1694(92)90105-5, 1992.

Neill, C., Chaves, J. E., Biggs, T., Deegan, L. A., Elsenbeer, H., Figueiredo, R. O., Germer, S., Johnson, M. S., Lehmann, J., Markewitz, D., and Piccolo, M. C.: Runoff sources and land cover change in the Amazon: An end-member mixing analysis from small watersheds, Biogeochemistry, 105, 7-18, https://doi.org/10.1007/s10533-011-9597-8, 2011.

Thurau, C., Kersting, K., Wahabzada, M., and Bauckhage, C.: Convex non-negative matrix factorization for massive datasets, Knowledge and Information Systems, 29, 457-478, https://doi.org/10.1007/s10115-010-0352-6, 2011.

Valder, J. F., Long, A. J., Davis, A. D., and Kenner, S. J.: Multivariate statistical approach to estimate mixing proportions for unknown end members, Journal of Hydrology, 460-461, 65-76, https://doi.org/10.1016/j.jhydrol.2012.06.037, 2012.

Wagstaff, K., Cardie, C., Rogers, S., and Schroedl, S.: Constrained k-means clustering with background knowledge, Proceedings of the Eighteenth International Conference on Machine Learning, 1, 677-584, 2001.

$\mathrm{Xu}$ Fei, E.: Example CHEMMA application code for the technical note, https://github.com/Estherrrrrxu/CHEMMA, https://doi.org/10.5281/zenodo.3841218, 2020.

Yang, L. and Hur, J.: Critical evaluation of spectroscopic indices for organic matter source tracing via end member mixing analysis based on two contrasting sources, Water Research, 59, 80-89, https://doi.org/10.1016/j.watres.2014.04.018, 2014. 
https://doi.org/10.5194/hess-2020-250

Preprint. Discussion started: 15 June 2020

(c) Author(s) 2020. CC BY 4.0 License.

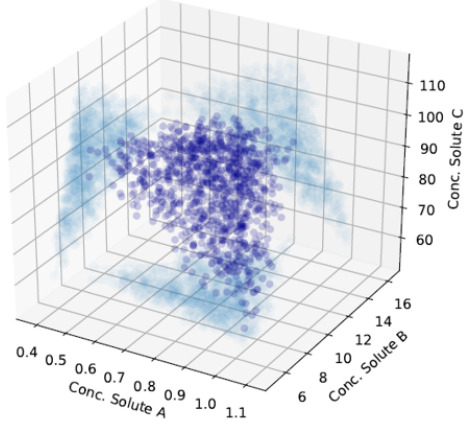

a)

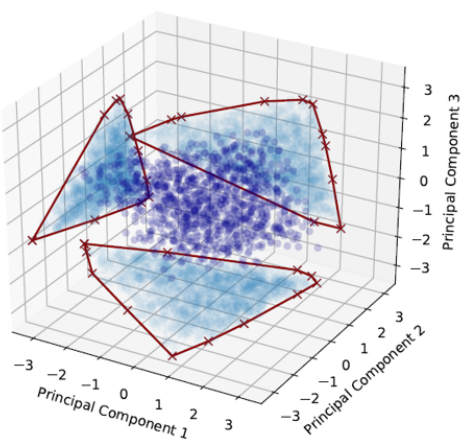

b)

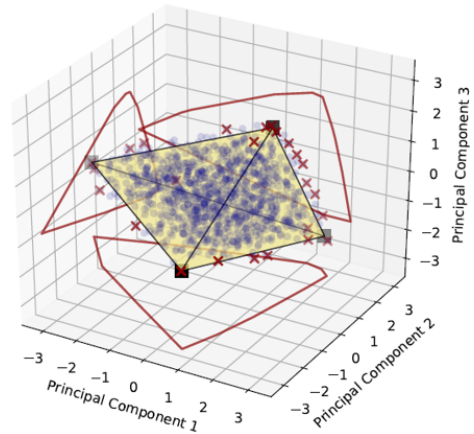

c)

Figure 1. Illustration of the CH-NMF algorithm. a) The standardized observations (dark blue) and its projection (light blue) on the observational space. b) The projected observations (dark blue) and its projection (light blue) on PC subspaces. The red crosses are the marked extreme points (S) that form a convex-hull (the red polygons) in each PC subspaces. c) Find the convex-hull (the black simplex) and its associated vertices (the $k$ vectors $\mathbf{x}_{e m i}$ ) in the PC space, such that the verticies are convex combinations of the extreme points $\mathbf{S}$, and the distance between the simplex and $\mathbf{S}$ is minimized. 
https://doi.org/10.5194/hess-2020-250

Preprint. Discussion started: 15 June 2020

(c) Author(s) 2020. CC BY 4.0 License.

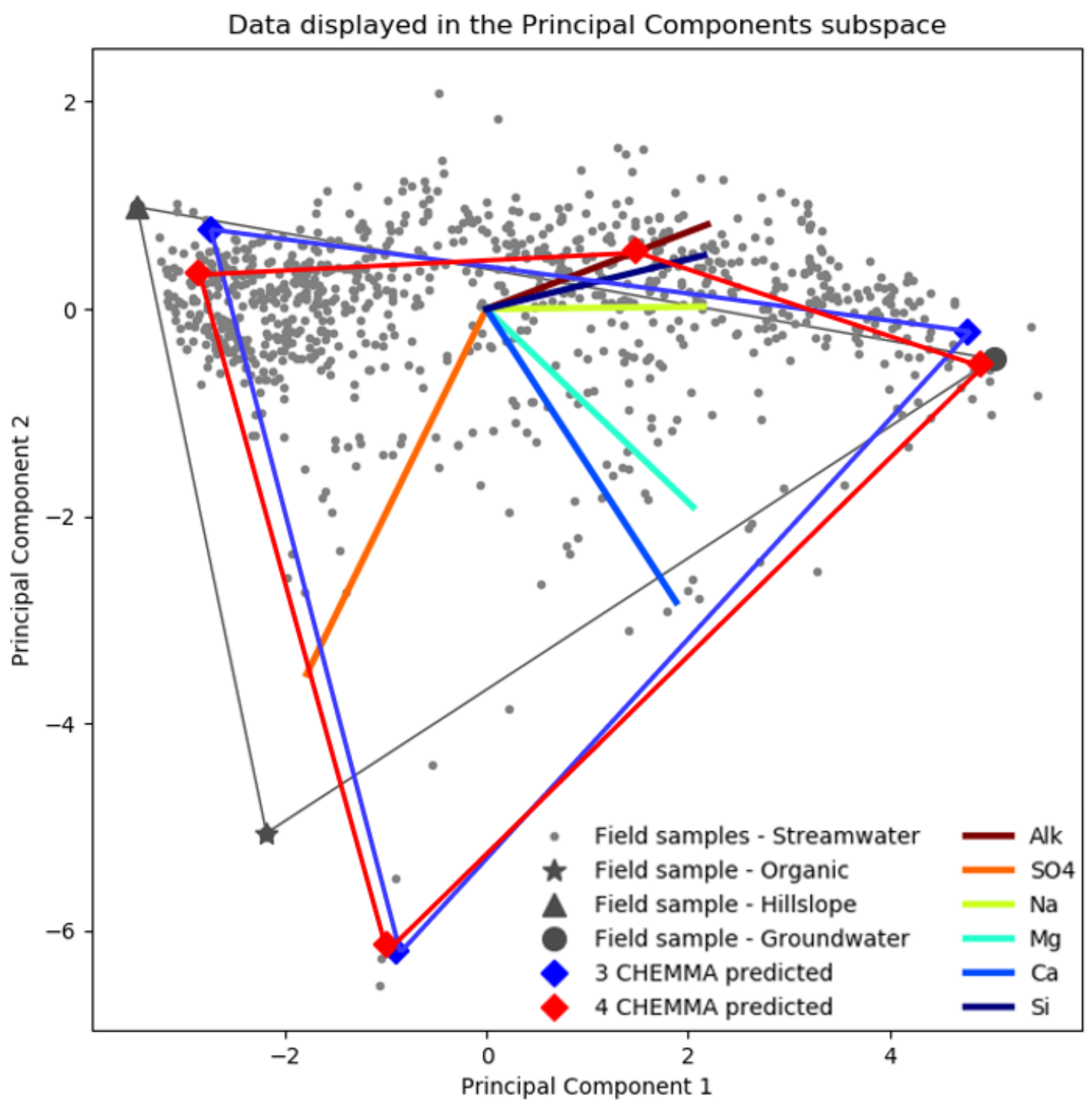

Figure 2. CHEMMA prediction (cluster centroids) for three end-member (blue squares) and four end-member (red squares) cases plotted in the PC2 vs. PC1 subspace. The colored lines that connect those predicted end-members indicate the convex hull formed by those endmembers. The observations (grey dots) inside of the convex-hull can be explained as linear combinations of the end-members. The colored lines in the center of the plot are the projected original solute axes in this PC subspace. 
https://doi.org/10.5194/hess-2020-250

Preprint. Discussion started: 15 June 2020

(c) Author(s) 2020. CC BY 4.0 License.
Hydrology and Earth System Sciences

Discussions

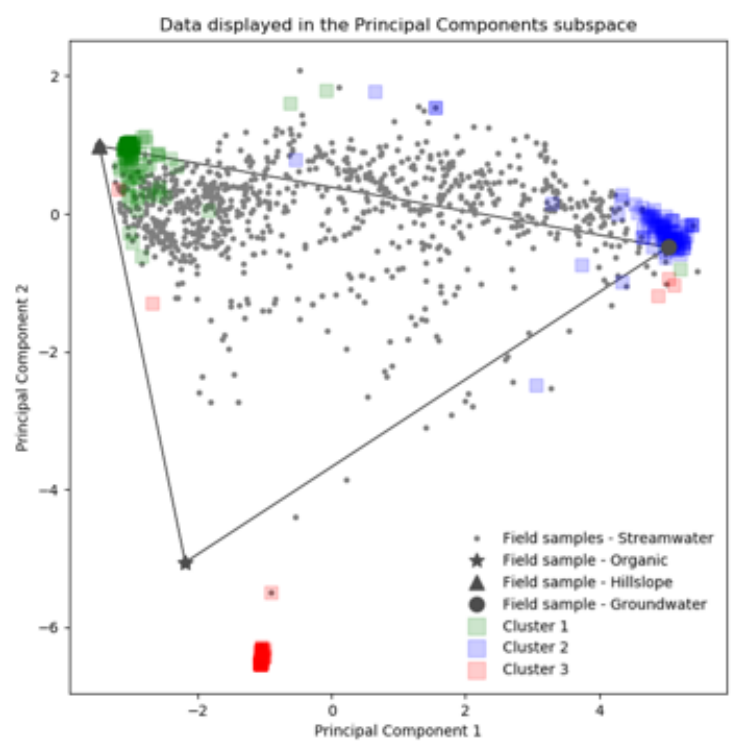

a)

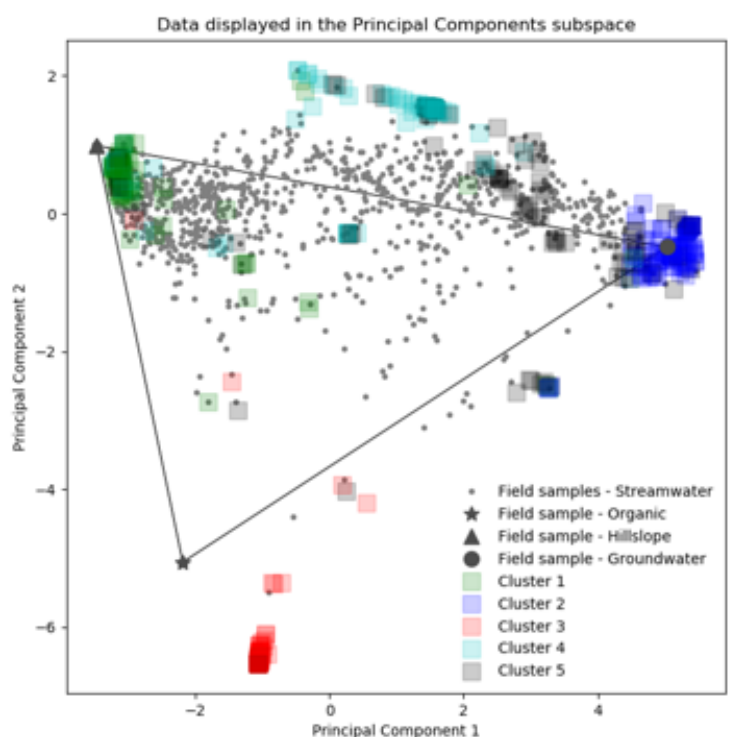

c)

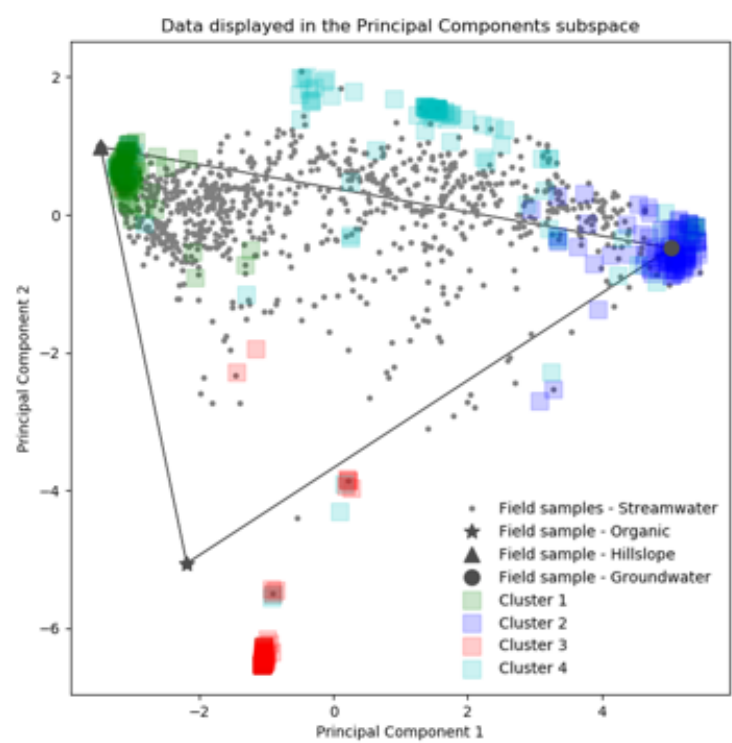

b)

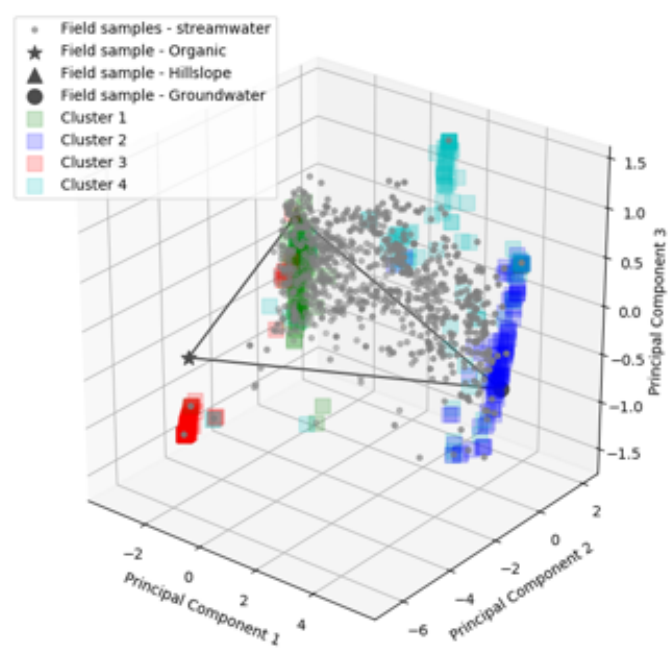

d)

Figure 3. 100 random initialized CH-NMF runs result for three (a), four (b and d), and five (c) end-member cases. a - c are in the 2D PC2 vs. PC1 subspaces. $d$ is in the 3D PC3 vs. PC2 vs. PC1 subspace. 


\begin{tabular}{|c|c|c|c|c|c|c|c|c|c|c|c|c|}
\hline \multirow{2}{*}{ Cluster } & \multicolumn{2}{|c|}{ Alkalinity } & \multicolumn{2}{|c|}{$\mathbf{S O}_{4}$} & \multicolumn{2}{|c|}{$\mathbf{N a}$} & \multicolumn{2}{|c|}{ Mg } & \multicolumn{2}{|c|}{$\mathrm{Ca}$} & \multicolumn{2}{|c|}{$\mathbf{S i}$} \\
\hline & Mean & St.dev & Mean & St.dev & Mean & St.dev & Mean & St.dev & Mean & St.dev & Mean & St.dev \\
\hline Red & 35.05 & 27.02 & 216.75 & 30.72 & 48.14 & 20.28 & 92.48 & 7.92 & 192.37 & 22.36 & 90.88 & 53.51 \\
\hline Blue & 348.04 & 12.16 & 14.11 & 2.82 & 214.87 & 21.88 & 90.35 & 4.64 & 151.26 & 9.93 & 405.86 & 23.55 \\
\hline Green & 33.43 & 32.27 & 77.45 & 12.60 & 44.70 & 20.01 & 32.03 & 5.84 & 47.14 & 10.75 & 100.34 & 55.85 \\
\hline Red & 32.86 & 12.33 & 219.71 & 17.57 & 46.66 & 9.91 & 93.50 & 2.44 & 193.92 & 15.11 & 87.25 & 28.64 \\
\hline Blue & 345.01 & 23.29 & 15.71 & 14.91 & 211.26 & 26.22 & 92.02 & 5.88 & 157.14 & 11.86 & 385.44 & 50.57 \\
\hline Green & 26.80 & 31.28 & 85.15 & 23.04 & 38.65 & 13.11 & 32.83 & 10.59 & 54.00 & 25.65 & 78.26 & 28.29 \\
\hline Cyan & 207.96 & 92.01 & 38.45 & 40.07 & 141.51 & 46.76 & 61.89 & 18.02 & 91.57 & 42.03 & 342.13 & 122.07 \\
\hline Red & 38.88 & 49.76 & 211.17 & 41.12 & 49.60 & 27.28 & 91.13 & 11.34 & 189.23 & 29.04 & 92.71 & 59.09 \\
\hline Blue & 344.76 & 21.77 & 15.88 & 14.39 & 211.90 & 30.95 & 92.44 & 5.63 & 158.67 & 12.07 & 390.34 & 40.03 \\
\hline Green & 29.62 & 33.35 & 85.37 & 13.38 & 42.52 & 17.68 & 33.40 & 6.83 & 52.32 & 16.99 & 84.20 & 29.38 \\
\hline Cyan & 171.83 & 77.99 & 40.85 & 33.32 & 123.60 & 44.11 & 54.77 & 15.08 & 75.69 & 29.17 & 329.06 & 138.29 \\
\hline Black & 253.45 & 107.65 & 44.10 & 47.45 & 161.55 & 58.00 & 75.81 & 17.47 & 125.51 & 38.38 & 278.05 & 123.41 \\
\hline
\end{tabular}

Table 1. The mean and standard deviation (st.dev) of each end-member cluster based on 100 random initialized CH-NMF runs. All values are in micromoles per liter. The cluster color indications correspond to Figure 3 a to c. 
https://doi.org/10.5194/hess-2020-250

Preprint. Discussion started: 15 June 2020

(c) Author(s) 2020. CC BY 4.0 License.

\begin{tabular}{ccccccc}
\hline Field individual samples & Alkalinity & SO $_{4}$ & Na & Mg & Ca & Si \\
\hline Organic & 37 & 214 & 23 & 78 & 151 & 60 \\
Groundwater & 370 & 7 & 169 & 97 & 162 & 422 \\
Hillslope & 9 & 89 & 46 & 22 & 32 & 90
\end{tabular}

Table 2. The median concentration of individual field measured end-members from Hooper and Christophersen (1992). All units are in micromoles per liter. 